\title{
Planar charged-particle trajectories in multipole magnetic fields
}

\author{
D. M. Willis ${ }^{1, *}$, A. R. Gardiner ${ }^{2}$, V. N. Davda ${ }^{1}$, V. J. Bone ${ }^{1,3}$ \\ ${ }^{1}$ Rutherford Appleton Laboratory, Chilton, Didcot, Oxon, OX11 0QX, UK \\ 2 School of Mathematical Sciences, University of Sussex, Falmer, Brighton, BN1 9QH, UK \\ ${ }^{3}$ School of Mathematical Studies, University of Portsmouth, Portsmouth, PO1 2EG, UK
}

Received: 3 January 1996 / Revised: 31 July 1996 / Accepted: 6 August 1996

\begin{abstract}
This paper provides a complete generalization of the classic result that the radius of curvature $(\rho)$ of a charged-particle trajectory confined to the equatorial plane of a magnetic dipole is directly proportional to the cube of the particle's equatorial distance $(\varpi)$ from the dipole (i.e. $\rho \propto \varpi^{3}$ ). Comparable results are derived for the radii of curvature of all possible planar chargedparticle trajectories in an individual static magnetic multipole of arbitrary order $m$ and degree $n$. Such trajectories arise wherever there exists a plane (or planes) such that the multipole magnetic field is locally perpendicular to this plane (or planes), everywhere apart from possibly at a set of magnetic neutral lines. Therefore planar trajectories exist in the equatorial plane of an axisymmetric $(m=0)$, or zonal, magnetic multipole, provided $n$ is odd: the radius of curvature varies directly as $\varpi^{n+2}$. This result reduces to the classic one in the case of a zonal magnetic dipole $(n=1)$. Planar trajectories exist in $2 m$ meridional planes in the case of the general tesseral $(0<m<n)$ magnetic multipole. These meridional planes are defined by the $2 m$ roots of the equation $\cos \left[m\left(\phi-\phi_{n}^{m}\right)\right]=0$, where $\phi_{n}^{m}=(1 / m)$ arctan $\left(h_{n}^{m} / g_{n}^{m}\right) ; g_{n}^{m}$ and $h_{n}^{m}$ denote the spherical harmonic coefficients. Equatorial planar trajectories also exist if $(n-m)$ is odd. The polar axis $(\theta=0, \pi)$ of a tesseral magnetic multipole is a magnetic neutral line if $m>1$. A further $2 m(n-m)$ neutral lines exist at the intersections of the $2 m$ meridional planes with the $(n-m)$ cones defined by the $(n-m)$ roots of the equation $P_{n}^{m}(\cos \theta)=0$ in the range $0<\theta<\pi$, where $P_{n}^{m}(\cos \theta)$ denotes the associated Legendre function. If $(n-m)$ is odd, one of these cones coincides with the equator and the magnetic field is then perpendicular to the equator everywhere apart from the $2 m$ equatorial neutral lines. The radius of curvature of an equatorial trajectory is directly proportional to $\varpi^{n+2}$ and inversely proportional to $\cos \left[m\left(\phi-\phi_{n}^{m}\right)\right]$. Since this last expression vanishes at the $2 m$ equatorial
\end{abstract}

\footnotetext{
* Also Visiting Reader in Physics, University of Sussex, Falmer, Brighton, BN1 9QH, UK

Correspondence to: D. M. Willis
}

neutral lines, the radius of curvature becomes infinitely large as the particle approaches any one of these neutral lines. The radius of curvature of a meridional trajectory is directly proportional to $r^{n+2}$, where $r$ denotes radial distance from the multipole, and inversely proportional to $P_{n}^{m}(\cos \theta) / \sin \theta$. Hence the radius of curvature becomes infinitely large if the particle approaches the polar magnetic neutral line $(m>1)$ or any one of the $2 m(n-m)$ neutral lines located at the intersections of the $2 m$ meridional planes with the $(n-m)$ cones. Illustrative particle trajectories, derived by stepwise numerical integration of the exact equations of particle motion, are presented for low-degree $(n \leq 3)$ magnetic multipoles. These computed particle trajectories clearly demonstrate the "non-adiabatic" scattering of charged particles at magnetic neutral lines. Brief comments are made on the different regions of phase space defined by regular and irregular trajectories.

\section{Introduction}

In his book The Polar Aurora, Störmer (1955) drew attention to an important property of the trajectories of those charged particles whose orbital motion is confined to the equatorial plane of a magnetic dipole. Every equatorial trajectory has the remarkable geometrical property that its radius of curvature at any point is proportional to the cube of its radial distance from the magnetic dipole. The prime purpose of this paper is to show that Störmer's result can be extended to planar charged-particle trajectories in general axisymmetric and non-axisymmetric multipole magnetic fields.

Mathematical investigations of charged-particle trajectories in an individual multipole magnetic field have important applications in studies of geomagnetically trapped radiation, the geographical distribution of precipitating auroral particles and the trajectories of cosmic rays near the Earth. Theoretical understanding of these three topics has been advanced significantly 
through detailed investigations of the motion of an isolated charged particle in a dipole magnetic field (Störmer, 1955; Vallarta, 1961; Alfvén and Fälthammar, 1963; Hess, 1968; Roederer, 1970; Walt, 1994; Walker and Russell, 1995). In fact, a dipole magnetic field has been used extensively in geomagnetism and magnetospheric physics as a first-order approximation to the contemporary geomagnetic field (Chapman and Bartels, 1940; Akasofu and Chapman, 1972). The dipole approximation to the geomagnetic field is often adequately accurate in the inner magnetosphere, where the distortion of the geomagnetic field by the flow of solarwind plasma is negligible. Extensions of the theory to the case of an isolated charged particle moving in an individual, non-dipolar, multipole magnetic field are important because such extensions have possible analogous applications during geomagnetic polarity reversals.

\section{Transitional-field models of geomagnetic polarity reversals}

Experimental studies of palaeomagnetic records spanning various polarity reversals have resulted in the development of several semi-quantitative models of the geomagnetic field during the transition interval, which typically lasts several thousand years (Fuller et al., 1979; Hoffman, 1982, 1983, 1985; Merrill and McElhinny, 1983; Jacobs, 1984; Clement and Kent, 1984 a,b, 1985; Bogue and Hoffman, 1987; Bogue and Merrill, 1992; McFadden and Merrill, 1995). However, palaeomagnetic evidence pertaining to one particular polarity reversal implies the existence of brief episodes of extraordinarily rapid field change of about six degrees per day (Coe et al., 1995). Initially, the experimental observations were interpreted as implying axisymmetric (or zonal), but non-dipolar, transitional magnetic fields, and many of the early models attempted to synthesize the observed transitional fields in terms of low-degree linear (or axial) multipoles. In most of the initial investigations (Hoffman, 1977, 1979; Dodson et al., 1978; Hoffman and Fuller, 1978; Fuller et al., 1979) it was claimed that the predominant component of the transitional magnetic field would be either a linear (axial) quadrupole or a linear (axial) octupole. Subsequently, Williams and Fuller (1981) developed a model of the transitional magnetic field based on the dominance of low-degree zonal harmonics or axial multipoles.

Although the initial interpretation of transition records did not exclude the possibility of non-axisymmetric magnetic-field components (Hoffman, 1979, 1981), several of the authors cited above maintained that the geomagnetic field was predominantly axisymmetric, but non-dipolar, during at least part of the Matuyama-Brunhes $(\mathrm{R} \rightarrow \mathrm{N})$ transition interval. More recent palaeomagnetic evidence has been interpreted as implying that the geomagnetic field was probably nonaxisymmetric, rather than axisymmetric, during at least some, if not all, polarity reversals (Clement and Kent, 1985, 1991; Hoffman, 1985; Prévot et al., 1985 a,b;
Theyer et al., 1985; Herrero-Bervera and Theyer, 1986; Bogue and Hoffman, 1987; Roperch and Chauvin, 1987; Valet et al., 1988, 1989, 1992; Laj et al., 1988; Weeks et al., 1988; Clement and Kent, 1991; Clement, 1991; Bogue and Merrill, 1992; McFadden and Merrill, 1995). Nevertheless, whenever there is some evidence for axial symmetry, as in the case of the Matuyama-Brunhes transition interval, its existence is sometimes more apparent during the initial (or decay) phase of the reversal process than the recovery phase (Hoffman, 1982, 1986; Mankinen et al., 1985; Prévot et al., 1985 a,b; Bogue and Hoffman, 1987; Roperch and Chauvin, 1987; Roperch and Duncan, 1990; Chauvin et al., 1990; Bogue and Merrill, 1992). This empirical evidence is in agreement with certain theoretical ideas on polarity reversals (Hide, 1981, 1982).

\section{Solar-terrestrial physics during geomagnetic polarity reversals}

Large changes in the geomagnetic field, over either historical or geological time-scales, would have produced significant changes in the nature of magnetospheric and solar-terrestrial conditions. Some of the more important possible changes have been explored in a series of pioneering papers by G. L. Siscoe and coworkers (Siscoe, 1976 a,b,c, 1979; Siscoe and Chen, 1975; Siscoe and Christopher, 1975; Siscoe and Crooker, 1976; Siscoe, Chen and Harel, 1976; Siscoe and Sibeck, 1980). Most of these investigations have been concerned primarily with the solar-terrestrial conditions that might be expected to arise during geomagnetic reversals or excursions. Similarly, Rishbeth (1985) has discussed the principal features that might exist in the terrestrial "palaeo-ionosphere" if the geomagnetic field were to assume either an axisymmetric or a non-axisymmetric quadrupolar form during a geomagnetic polarity reversal.

As a useful theoretical preliminary to a detailed study of magnetospheric, ionospheric and cosmic-ray physics during geomagnetic polarity reversals, Willis and Young (1987) derived an exact equation for the magnetic field lines of a general axisymmetric magnetic multipole of arbitrary degree (n). Subsequently, Jeffreys (1988) presented an alternative, and somewhat simpler, mathematical derivation of the equation for the field lines of a single axisymmetric multipole and Backus (1988) generalized this result to the case of an arbitrary linear combination of axisymmetric multipoles. In a special extension to non-axisymmetric magnetic fields, Willis and Gardiner (1988) derived exact equations for the magnetic field lines of both symmetric sectorial $(m=n)$ and anti-symmetric sectorial $(m=n-1)$ magnetic multipoles of arbitrary degree $(n)$.

As implied in the Introduction, major changes in the configuration of the geomagnetic field during polarity reversals would almost certainly lead to dramatic changes in geomagnetically trapped radiation, the geographical distribution of precipitating auroral parti- 
cles and the distribution of cosmic rays impinging on the Earth's upper atmosphere. Willis and Gardiner (1988) inferred that planar (two-dimensional) charged-particle trajectories would exist for both symmetric sectorial $(m=n)$ and anti-symmetric sectorial $(m=n-1)$ multipole magnetic fields. This inference was based entirely on the geometrical configuration of the magnetic field lines for such multipoles. The purpose of this paper is to prove rigorously that planar trajectories exist for charged-particle motion in most individual axisymmetric and non-axisymmetric multipole magnetic fields. Therefore, the theory developed here provides a complete generalization of the classic result for charged-particle motion in the equatorial plane of a magnetic dipole (Störmer, 1955). However, it should be emphasized at the outset that the discussion is restricted to two-dimensional (planar) trajectories, and no attempt is made in this paper to determine the allowed and forbidden regions for full three-dimensional particle motion.

\section{The magnetic field of a general multipole}

Following the procedure adopted in previous papers (Willis and Young, 1987; Willis and Gardiner, 1988), it is convenient to derive the equations that define the magnetic field of a general magnetic multipole from the general spherical harmonic expansion of the Earth's main magnetic field.

At any given instant of time (epoch) the external scalar potential, $V(r, \theta, \phi)$, of the Earth's main magnetic field, which is of internal origin, can be expressed in the following form (Chapman and Bartels, 1940; Roederer, 1972; Stern, 1976):

$V(r, \theta, \phi)=\sum_{n=1}^{\infty} V_{n}(r, \theta, \phi)$,

where

$$
\begin{aligned}
V_{n}(r, \theta, \phi)= & R_{\mathrm{E}}\left(R_{\mathrm{E}} / r\right)^{n+1} \\
& \times \sum_{m=0}^{n} c_{n}^{m} \cos \left[m\left(\phi-\phi_{n}^{m}\right)\right] P_{n}^{m}(\cos \theta), \\
c_{n}^{m}=\mid\left[\left(g_{n}^{m}\right)^{2}+\right. & \left.\left(h_{n}^{m}\right)^{2}\right]^{1 / 2} \mid, c_{n}^{0}=g_{n}^{0}\left(h_{n}^{0} \equiv 0\right)
\end{aligned}
$$

and

$$
\phi_{n}^{m}=(1 / m) \arctan \left(h_{n}^{m} / g_{n}^{m}\right)=(1 / m) \arcsin \left(h_{n}^{m} / c_{n}^{m}\right) .
$$

In this representation of the geomagnetic field, positions on the surface of the Earth are specified in terms of spherical polar coordinates $(r, \theta, \phi)$ with origin $\mathrm{O}$ at the centre of the Earth; $r$ is the radial distance $\left(r \geq R_{\mathrm{E}}\right), \theta$ is the geographic colatitude with the north geographic pole at $\theta=0$, and $\phi$ is the geographic longitude measured east from Greenwich. The radius of the reference sphere, $r=R_{\mathrm{E}}$, is taken to be the mean radius of the Earth $(6371.2 \mathrm{~km}) ; P_{n}^{m}(\cos \theta)$ is Schmidt's partially (or quasi-) normalized associated Legendre function of order $m$ and degree $n$ (where $m$ and $n$ are integers); $g_{n}^{m}$ and $h_{n}^{m}$ are the spherical harmonic (or Schmidt) coefficients for the particular epoch considered; and all physical quantities are measured in SI units. In this paper the definition of (magnetic) scalar potential $(V)$ is such that the spherical harmonic coefficients $g_{n}^{m}$ and $h_{n}^{m}$ have the dimensions of magnetic induction (i.e. $\boldsymbol{B}=-\operatorname{grad} V$ ).

The total scalar potential defined by Eqs. 1 and 2 is just a double summation over all scalar potentials of the form

$V_{n}^{m}=c_{n}^{m} R_{\mathrm{E}}\left(R_{\mathrm{E}} / r\right)^{n+1} \cos \left[m\left(\phi-\phi_{n}^{m}\right)\right] P_{n}^{m}(\cos \theta)$,

each of which defines an individual magnetic multipole of order $m$ and degree $n(0 \leq m \leq n)$. The parameter $c_{n}^{m}$ represents the "total strength" of this multipole and the parameter $\phi_{n}^{m}$ denotes the phase angle (with respect to the reference meridian $\phi=0$ ). In the special case of an axisymmetric (zonal) magnetic multipole, $m=0$, and it then follows from Eqs. 3 and 4 that Eq. 5 reduces to the form

$V_{n}^{0}=g_{n}^{0} R_{\mathrm{E}}\left(R_{\mathrm{E}} / r\right)^{n+1} P_{n}^{0}(\cos \theta)$.

The magnetic-field components of the general magnetic multipole can be found from the equation $\boldsymbol{B}=$ $-\operatorname{grad}\left(V_{n}^{m}\right)$, with $V_{n}^{m}$ defined by Eq. 5. In spherical polar coordinates, the three magnetic-field components are

$$
\begin{aligned}
& B_{r}=(n+1) c_{n}^{m}\left(R_{\mathrm{E}} / r\right)^{n+2} \cos \left[m\left(\phi-\phi_{n}^{m}\right)\right] P_{n}^{m}(\cos \theta),(7) \\
& B_{\theta}=-c_{n}^{m}\left(R_{\mathrm{E}} / r\right)^{n+2} \cos \left[m\left(\phi-\phi_{n}^{m}\right)\right] \frac{d P_{n}^{m}(\cos \theta)}{d \theta} \\
& B_{\phi}=m c_{n}^{m}\left(R_{\mathrm{E}} / r\right)^{n+2} \sin \left[m\left(\phi-\phi_{n}^{m}\right)\right] \frac{P_{n}^{m}(\cos \theta)}{\sin \theta}
\end{aligned}
$$

In the special case of a zonal magnetic multipole, $m=0, c_{n}^{0}=g_{n}^{0}\left(h_{n}^{0} \equiv 0\right)$ and $d P_{n}^{0}(\cos \theta) / d \theta=-[n(n+1)$ $/ 2]^{1 / 2} P_{n}^{1}(\cos \theta)$ this last equation is presented by Chapman and Bartels (1940; Chap. XVII, Eq. 55). Equations 7-9 then reduce to the pair of equations

$$
B_{r}=(n+1) g_{n}^{0}\left(R_{\mathrm{E}} / r\right)^{n+2} P_{n}^{0}(\cos \theta)
$$

and

$B_{\theta}=[n(n+1) / 2]^{1 / 2} g_{n}^{0}\left(R_{\mathrm{E}} / r\right)^{n+2} P_{n}^{1}(\cos \theta)$.

The magnetic-field-line configuration of this general axisymmetric magnetic multipole has been considered in detail by Willis and Young (1987). Moreover, in the special cases $m=n$ and $m=n-1$, the series expansion for $P_{n}^{m}(\cos \theta)$ given by Chapman and Bartels (1940; Chap. XVII, Eqs. 10 and 20) can be used to reduce the magnetic-field components to purely trigonometric forms. Then Eqs. 7-9 simplify to the two sets of three equations considered by Willis and Gardiner (1988) in their discussion of the magnetic-field-line configurations of symmetric sectorial $(m=n)$ and anti-symmetric sectorial $(m=n-1)$ magnetic multipoles.

In the following discussion of the motion of an isolated charged particle in an individual multipole magnetic field, it is advantageous to refer the particle motion to a right-handed system of rectangular Cartesian coordinates $\mathrm{O}(x, y, z)$, with origin $\mathrm{O}$ at the centre of 
the Earth and $z$-axis coincident with the polar axis $(\theta=0, \pi)$ of the system of spherical polar coordinates. The magnetic-field components in Cartesian coordinates are related to those in spherical polar coordinates by the three equations

$$
\begin{aligned}
& B_{x}=B_{r} \sin \theta \cos \phi+B_{\theta} \cos \theta \cos \phi-B_{\phi} \sin \phi, \\
& B_{y}=B_{r} \sin \theta \sin \phi+B_{\theta} \cos \theta \sin \phi+B_{\phi} \cos \phi, \\
& B_{z}=B_{r} \cos \theta-B_{\theta} \sin \theta .
\end{aligned}
$$

\section{Motion of a charged particle in a general magnetic field}

It is convenient to define in this section certain general results for the motion of an isolated charged particle in a magnetic field. The general results presented in this and the previous sections can then be used to consider planar charged-particle trajectories in particular multipole magnetic fields.

If radiation losses are neglected, the relativistic equation of motion of an isolated charged particle of rest mass $m_{0}$, charge $Q e$ (where $e$ denotes the magnitude of the charge on an electron) and velocity $\boldsymbol{v}$ moving in a static magnetic induction $\boldsymbol{B}$ is (Alfvén and Fälthammar, 1963; Northrop, 1963)

$\frac{d}{d t}\left(\gamma m_{0} \boldsymbol{v}\right)=Q e(\boldsymbol{v} \times \mathbf{B})$,

where $\gamma=\left(1-|v|^{2} / c^{2}\right)^{-1 / 2}$ and $c$ denotes the speed of light. It is clear from the form of Eq. 15 that the speed $V$ $(=|v|)$ of the particle remains constant, and hence it is possible to write

$s=V t$,

where $s$ denotes arc length along the particle trajectory, measured from a fixed point.

Referred to the right-handed system of rectangular Cartesian coordinates $\mathrm{O}(x, y, z)$ introduced in Sect. 4, the three components of Eq. 15 can then be expressed in the form (Störmer, 1955; Sandström, 1965)

$$
\begin{aligned}
& \frac{d^{2} x}{d s^{2}}=\left(\frac{Q e}{\gamma m_{0} V}\right)\left[B_{z} \frac{d y}{d s}-B_{y} \frac{d z}{d s}\right], \\
& \frac{d^{2} y}{d s^{2}}=\left(\frac{Q e}{\gamma m_{0} V}\right)\left[B_{x} \frac{d z}{d s}-B_{z} \frac{d x}{d s}\right], \\
& \frac{d^{2} z}{d s^{2}}=\left(\frac{Q e}{\gamma m_{0} V}\right)\left[B_{y} \frac{d x}{d s}-B_{x} \frac{d y}{d s}\right],
\end{aligned}
$$

where $\boldsymbol{B}=\left(B_{x}, B_{y}, B_{z}\right), \boldsymbol{v}=(u, v, w)$ and $u=d x / d t=$ $V d x / d s$, etc. Since the constant speed $V$ of the particle is given by

$$
\begin{aligned}
V^{2} & =u^{2}+v^{2}+w^{2} \\
& =(d x / d t)^{2}+(d y / d t)^{2}+(d z / d t)^{2},
\end{aligned}
$$

it follows that

$$
(d x / d s)^{2}+(d y / d s)^{2}+(d z / d s)^{2}=1 .
$$

The general equation for the radius of curvature, $\rho$, of the trajectory of an isolated charged particle is given by

$$
1 / \rho^{2}=\left(d^{2} x / d s^{2}\right)^{2}+\left(d^{2} y / d s^{2}\right)^{2}+\left(d^{2} z / d s^{2}\right)^{2} .
$$

For any specific multipole magnetic field $\left(B_{x}, B_{y}, B_{z}\right)$, Eqs. $17-19$ can be substituted into Eq. 22 to yield the corresponding specific expression for the radius of curvature of the particle trajectory.

\section{Equatorial trajectories in zonal multipole magnetic fields}

For a zonal $(m=0)$, or axisymmetric, multipole magnetic field, $B_{\phi}=0$ and the other two magnetic-field components, $B_{r}$ and $B_{\theta}$, are defined by Eqs. 10 and 11, respectively. In the equatorial plane $(\theta=\pi / 2)$, Schmidt's partially normalized associated Legendre functions $\mathrm{P}_{n}^{0}$ $(\cos \theta)$ and $P_{n}^{1}(\cos \theta)$ can be expressed in the following trigonometric forms (Erdélyi et al., 1953; Vol. I, Sect. 3.4, amended Eq. 20)

$P_{n}^{0}(0)=\pi^{-1 / 2} \cos (n \pi / 2) \Gamma(n / 2+1 / 2) / \Gamma(n / 2+1)$

and

$$
\begin{aligned}
P_{n}^{1}(0)= & -2[2 / n(n+1)]^{1 / 2} \pi^{-1 / 2} \\
& \times \cos [(n+1) \pi / 2] \Gamma(n / 2+1) / \Gamma(n / 2+1 / 2),
\end{aligned}
$$

where $\Gamma$ denotes the gamma function. It is clear from Eqs. $10,11,23$ and 24 that $B_{r}=0, B_{\theta} \neq 0$ in the equatorial plane if $n$ is odd, whereas $B_{r} \neq 0, B_{\theta}=0$ in the equatorial plane if $n$ is even. Therefore, the magnetic field lines of an axisymmetric magnetic multipole are locally perpendicular to the equatorial plane if $n$ is odd and lie in the equatorial plane if $n$ is even. This theoretical result is completely consistent with the illustrative magnetic-field-line configurations for lowdegree $(1 \leq n \leq 4)$ axisymmetric magnetic multipoles presented in Fig. 1 of the paper by Willis and Young (1987).

From the foregoing discussion, it is intuitively clear on physical grounds that (planar) equatorial trajectories exist in zonal multipole magnetic fields if the degree $(n)$ of the multipole is odd. This result can be proved rigorously as follows. If $n$ is odd $\left(B_{r}=0, B_{\theta} \neq 0\right.$ if $\theta=\pi / 2$ and $\left.B_{\phi} \equiv 0\right)$, substituting Eqs. 10 and 11 into Eqs. 12 14, and using Eqs. 23 and 24, yields the following equations for the Cartesian components of the magnetic field in the equatorial plane $(\theta=\pi / 2)$ of an axisymmetric multipole

$B_{x}=B_{y}=0$

and

$B_{z}=-[n(n+1) / 2]^{1 / 2} g_{n}^{0} R_{\mathrm{E}}^{n+2} P_{n}^{1}(0) /\left(x^{2}+y^{2}\right)^{(n+2) / 2}$,

since $z=0$ if $\theta=\pi / 2$ and $P_{n}^{0}(0)=0$ if $n$ is odd. If Eqs. 25 and 26 are substituted into Eqs. 17-19, the three differential equations that define the motion of a charged particle in the equatorial plane of an axisymmetric magnetic multipole become 


$$
\begin{aligned}
& \frac{d^{2} x}{d s^{2}}=-\left(\frac{Q e}{\gamma m_{0} V}\right)\left[\frac{[n(n+1) / 2]^{1 / 2} g_{n}^{0} R_{\mathrm{E}}^{n+2} P_{n}^{1}(0)}{\left(x^{2}+y^{2}\right)^{(n+2) / 2}}\right] \frac{d y}{d s}, \\
& \frac{d^{2} y}{d s^{2}}=+\left(\frac{Q e}{\gamma m_{0} V}\right)\left[\frac{[n(n+1) / 2]^{1 / 2} g_{n}^{0} R_{\mathrm{E}}^{n+2} P_{n}^{1}(0)}{\left(x^{2}+y^{2}\right)^{(n+2) / 2}}\right] \frac{d x}{d s},
\end{aligned}
$$

$\frac{d^{2} z}{d s^{2}}=0$

Equation 29 confirms that the charged particle remains in the equatorial plane if $d z / d s=0$, that is if $w=0$.

If Eqs. 27-29 are now substituted into Eq. 22, the radius of curvature of the equatorial particle trajectory is given by

$$
\begin{aligned}
\frac{1}{\rho^{2}}= & \left(\frac{Q e g_{n}^{0}}{\gamma m_{0} V}\right)^{2}\left[\frac{[n(n+1) / 2]\left[P_{n}^{1}(0)\right]^{2}}{\left[\left(x^{2}+y^{2}\right) / R_{\mathrm{E}}^{2}\right]^{n+2}}\right] \\
& \times\left[\left(\frac{d x}{d s}\right)^{2}+\left(\frac{d y}{d s}\right)^{2}\right] .
\end{aligned}
$$

Writing $\varpi^{2}=x^{2}+y^{2} \quad(z=0)$ and remembering that $(d x / d s)^{2}+(d y / d s)^{2}=1(d z / d s=0)$ from Eq. 21, and $P_{n}^{1}(0)$ is defined by Eq. 24, this last equation can be simplified to the form

$\rho=\left(\frac{\gamma m_{0} V}{\operatorname{Qeg}_{n}^{0}}\right)\left[\frac{\pi^{1 / 2} \Gamma(n / 2+1 / 2)}{\Gamma(n / 2+1)}\right]\left(\frac{\varpi}{R_{\mathrm{E}}}\right)^{n+2}$,

provided the degree $(n)$ of the axisymmetric multipole is odd so that $\cos ^{2}[(n+1) \pi / 2]=1$. For a dipole magnetic field $(n=1)$, Eq. 31 reduces to the form

$\rho=\left(\frac{\gamma m_{0} V}{Q e g_{1}^{0}}\right)\left(\frac{\varpi}{R_{\mathrm{E}}}\right)^{3}$

since $\Gamma(1)=1$ and $\Gamma(3 / 2)=(1 / 2) \pi^{1 / 2}$.

It follows from Eq. 32 that the radius of curvature of the trajectory of a charged particle moving in the equatorial plane of a magnetic dipole $(n=1)$ is proportional to the cube of the equatorial distance of the particle from the dipole $\left(\rho \propto \varpi^{3}\right)$. This result is essentially the same as that given by Störmer (1955; cf., his Eq. 8.6). Equation 31 is the extension of Störmer's result to the case of a charged particle moving in the equatorial plane of an axisymmetric magnetic multipole of odd degree $n$, so that $\rho$ is directly proportional to $\varpi^{n+2}$ in the general case.

\section{Meridional trajectories in sectorial multipole magnetic fields}

The magnetic-field configurations of both symmetric sectorial $(m=n)$ and anti-symmetric sectorial ( $m=n-1)$ magnetic multipoles have been considered in detail by Willis and Gardiner (1988). As shown by these authors, the magnetic-field components of a symmetric sectorial $(m=n)$ magnetic multipole can be expressed in the trigonometric form

$B_{r}=(n+1) C_{n}^{n} r^{-(n+2)} \sin ^{n} \theta \cos \left[n\left(\phi-\phi_{n}^{n}\right)\right]$,

$$
\begin{aligned}
& B_{\theta}=-n C_{n}^{n} r^{-(n+2)} \sin ^{n-1} \theta \cos \theta \cos \left[n\left(\phi-\phi_{n}^{n}\right)\right], \\
& B_{\phi}=n C_{n}^{n} r^{-(n+2)} \sin ^{n-1} \theta \sin \left[n\left(\phi-\phi_{n}^{n}\right)\right],
\end{aligned}
$$

where the parameter $C_{n}^{n}$ is defined by the equation

$C_{n}^{n}=\frac{[2(2 n) !]^{1 / 2}}{2^{n} n !} c_{n}^{n} R_{\mathrm{E}}^{n+2}$

and $c_{n}^{n}$ is defined by Eq. 3 .

As noted by Willis and Gardiner (1988), the magnetic field of a symmetric sectorial $(m=n)$ multipole is locally perpendicular to the $2 n$ meridional planes $\phi=\phi_{n}^{n}+$ $(2 k+1) \pi / 2 n=\phi_{k}^{*}$, where $k=0,1, \ldots, 2 n-1$; that is, $B_{r}$ $=B_{\theta}=0, B_{\phi} \neq 0$ everywhere in these planes apart from the polar axis $(\theta=0, \pi)$, which is a neutral line if $n>1$. The magnetic-field components in these $2 n$ meridional planes are given by.7

$B_{r}=B_{\theta}=0$

and

$B_{\phi}=(-1)^{k} n C_{n}^{n} r^{-(n+2)} \sin ^{n-1} \theta$.

Substituting these components into Eqs. 12-14 yields the following equations for the Cartesian components of the magnetic field in the $2 n$ meridional planes $\phi=\phi_{k}^{*}$ :

$B_{x}=-(-1)^{k} n C_{n}^{n} r^{-(n+2)} \sin ^{n-1} \theta \sin \phi_{k}^{*}$,

$B_{y}=+(-1)^{k} n C_{n}^{n} r^{-(n+2)} \sin ^{n-1} \theta \cos \phi_{k}^{*}$,

$B_{z}=0$.

If Eqs. 39-41 are substituted into Eqs. 17-19, the three differential equations that define the motion of a charged particle in one of the $2 n$ meridional planes $\phi=\phi_{k}^{*}$ become

$$
\begin{aligned}
\frac{d^{2} x}{d s^{2}}= & -\left(\frac{Q e}{\gamma m_{0} V}\right)\left[(-1)^{k} n C_{n}^{n} r^{-(n+2)} \sin ^{n-1} \theta\right] \\
& \times \cos \phi_{k}^{*} \frac{d z}{d s}, \\
\frac{d^{2} y}{d s^{2}}= & -\left(\frac{Q e}{\gamma m_{0} V}\right)\left[(-1)^{k} n C_{n}^{n} r^{-(n+2)} \sin ^{n-1} \theta\right] \\
& \times \sin \phi_{k}^{*} \frac{d z}{d s}, \\
\frac{d^{2} z}{d s^{2}}= & +\left(\frac{Q e}{\gamma m_{0} V}\right)\left[(-1)^{k} n C_{n}^{n} r^{-(n+2)} \sin ^{n-1} \theta\right] \\
& \times\left[\cos \phi_{k}^{*} \frac{d x}{d s}+\sin \phi_{k}^{*} \frac{d y}{d s}\right] .
\end{aligned}
$$

It follows from Eqs. 42 and 43 that

$\cos \phi_{k}^{*} d^{2} y / d s^{2}-\sin \phi_{k}^{*} d^{2} x / d s^{2}=0$,

which implies that the particle remains in the plane $\phi=\phi_{k}^{*}$ if

$\cos \phi_{k}^{*} d y / d s-\sin \phi_{k}^{*} d x / d s=0$.

If Eqs. 42-44 are substituted into Eq. 22, and Eq. 46 is used to simplify the resulting expression, it follows that $\rho$ is given by 


$$
\begin{aligned}
\frac{1}{\rho^{2}}= & \left(\frac{Q e}{\gamma m_{0} V}\right)^{2}\left[(-1)^{k} n C_{n}^{n} r^{-(n+2)} \sin ^{n-1} \theta\right]^{2} \\
& \times\left[\left(\frac{d x}{d s}\right)^{2}+\left(\frac{d y}{d s}\right)^{2}+\left(\frac{d z}{d s}\right)^{2}\right] .
\end{aligned}
$$

Using Eqs. 21 and 36, the expression for the radius of curvature of a charged particle moving in one of the $2 n$ meridional planes, $\phi=\phi_{n}^{n}+(2 k+1) \pi / 2 n=\phi_{k}^{*}$, where $k=0,1,2, \ldots, 2 n-1$, becomes

$$
\begin{aligned}
\rho= & \left(\frac{\gamma m_{0} V}{Q e c_{n}^{n}}\right)\left[\frac{2^{n}(n-1) !}{[2(2 n) !]^{1 / 2}}\right] \\
& \times \frac{\left[\left(x^{2}+y^{2}+z^{2}\right) / R_{\mathrm{E}}^{2}\right]^{(2 n+1) / 2}}{\left[\left(x^{2}+y^{2}\right) / R_{\mathrm{E}}^{2}\right]^{(n-1) / 2}},
\end{aligned}
$$

since $r^{2}=x^{2}+y^{2}+z^{2}$ and $\sin \theta=\left(x^{2}+y^{2}\right)^{1 / 2} /\left(x^{2}+y^{2}\right.$ $\left.+z^{2}\right)^{1 / 2}$.

It follows from Eq. 48 that the radius of curvature of a charged particle moving in one of the $2 n$ special meridional planes $\phi=\phi_{k}^{*}(k=0,1,2, \ldots, 2 n-1)$ of a symmetric sectorial magnetic multipole is directly proportional to $r^{2 n+1}$ and inversely proportional to $\varpi^{n-1}$, where $r$ denotes radial distance from the multipole and $\varpi$ denotes perpendicular distance from the polar axis $(\theta=$ $0, \pi)$. The polar axis is a neutral line if $n>1$ (Willis and Gardiner, 1988) and in this case Eq. 48 implies that $\rho \rightarrow$ $\infty$ as $\varpi=\left(x^{2}+y^{2}\right)^{1 / 2} \rightarrow 0$. This result confirms the physically intuitive conclusion that the radius of curvature becomes infinitely large as the particle approaches the polar neutral line $(n>1)$.

In the degenerate case $m=n=1$, which corresponds to a dipole lying in the equatorial plane (Roederer, 1972; Willis and Gardiner, 1988), Eq. 48 reduces to the form

$\rho=\left(\frac{\gamma m_{0} V}{Q e c_{1}^{1}}\right)\left(\frac{r}{R_{\mathrm{E}}}\right)^{3}$,

where the equality $c_{1}^{1}=\left[\left(g_{1}^{1}\right)^{2}+\left(h_{1}^{1}\right)^{2}\right]^{1 / 2}$ specifies the strength of the dipole. This equation is the same as Eq. 32 apart from the value of the spherical harmonic coefficient (i.e. $c_{1}^{1}$ replaces $g_{1}^{0}$ ). In this degenerate case the two special meridional planes $\phi=\phi_{1}^{1}+\pi / 2$ and $\phi=\phi_{1}^{1}+3 \pi / 2(k=0,1)$ define the dipole magnetic equatorial plane in which the particle trajectory lies, which accounts for the essential equivalence of Eqs. 32 and 49.

\section{Planar trajectories in tesseral multipole magnetic fields}

\subsection{Equatorial and meridional magnetic-field components}

The magnetic-field components of the general tesseral $(0<m<n)$ magnetic multipole are defined by Eqs. 7-9. It follows from the form of these three Equations, and Eq. 7 in the paper by Willis and Gardiner (1988), that the polar axis $(\theta=0, \pi)$ is a neutral line $\left(B_{r}=B_{\theta}=B_{\phi}\right.$ $=0)$ if $m>1$. In addition, neutral lines exist at the intersections of the $2 m$ meridional planes $\phi=\phi_{n}^{m}+$ $(2 k+1) \pi / 2 m=\phi_{k}$, where $k=0,1,2, \ldots, 2 m-1$, with the $(n-m)$ cones $\theta=\theta_{i}$, where $\theta_{i}(1 \leq i \leq n-m)$ denote the $(n-m)$ different roots of the equation $P_{n}^{m}(\cos \theta)=0$ in the range $0<\theta<\pi$ (Chapman and Bartels, 1940; Chap. XVII, Sect. 17.6). Therefore, in the general case there are $2 m(n-m)$ neutral lines apart from the polar axis. If $(n-m)$ is odd, one of the $(n-m)$ zeros of $P_{n}^{m}$ $(\cos \theta)$ occurs at the equator $(\theta=\pi / 2)$, so that there are then $2 m$ equatorial neutral lines.

It follows from the preceding discussion that if $(n-m)$ is odd the magnetic field of a tesseral magnetic multipole is locally perpendicular to the equatorial plane $(\theta=\pi / 2)$; that is $B_{r}=B_{\phi}=0, \quad B_{\theta} \neq 0$ everywhere in the equatorial plane apart from the $2 \mathrm{~m}$ equatorial neutral lines. Similarly, the magnetic field of a tesseral magnetic multipole is locally perpendicular to the $2 m$ meridional planes $\phi=\phi_{n}^{m}+(2 k+1) \pi / 2 m=\phi_{k}^{*}$ $(k=0,1,2, \ldots, 2 m-1)$; that is $B_{r}=B_{\theta}=0, B_{\phi} \neq 0$ everywhere in these planes apart from the $2 m(n-m)$ neutral lines lying on the $(n-m)$ cones $\theta=\theta_{i} \quad(i=1$, $2,3, \ldots, n-m)$ and also the polar axis, which is a neutral line if $m>1$. Therefore, if $(n-m)$ is odd, the magnetic-field components in the equatorial plane $(\theta=\pi / 2$ are given by

$B_{r}=B_{\phi}=0$

and

$$
\begin{aligned}
B_{\theta}= & -c_{n}^{m}\left(R_{\mathrm{E}} / r\right)^{n+2} \cos \left[m\left(\phi-\phi_{n}^{m}\right)\right] \\
& \times\left[d P_{n}^{m}(\cos \theta) / d \theta\right]_{\theta=\pi / 2},
\end{aligned}
$$

whereas the magnetic-field components in the $2 m$ meridional planes $\phi=\phi_{k}^{*}$ are given by

$B_{r}=B_{\theta}=0$

and

$B_{\phi}=(-1)^{k} m c_{n}^{m}\left(R_{\mathrm{E}} / r\right)^{n+2}\left[P_{n}^{m}(\cos \theta) / \sin \theta\right]$.

Substituting Eqs. 50 and 51 into Eqs. 12-14 yields the following equations for the Cartesian components of the magnetic field in the equatorial plane $(\theta=\pi / 2, z=0$, $\left.\varpi^{2}=x^{2}+y^{2}\right)$ :

$B_{x}=B_{y}=0$

and

$$
\begin{aligned}
B_{z}= & c_{n}^{m}\left(R_{\mathrm{E}} / \varpi\right)^{n+2} \cos \left[m\left(\phi-\phi_{n}^{m}\right)\right] \\
& \times\left[d P_{n}^{m}(\cos \theta) / d \theta\right]_{\theta=\pi / 2} .
\end{aligned}
$$

Similarly, substituting Eqs. 52 and 53 into Eqs. 12-14 yields the following equations for the Cartesian components of the magnetic field in the $2 \mathrm{~m}$ meridional planes $\phi=\phi_{k}^{*}(k=0,1,2, \ldots, 2 m-1)$ :

$$
\begin{aligned}
B_{x} & =-(-1)^{k} m c_{n}^{m}\left(R_{\mathrm{E}} / r\right)^{n+2}\left[P_{n}^{m}(\cos \theta) / \sin \theta\right] \sin \phi_{k}^{*} \\
B_{y} & =+(-1)^{k} m c_{n}^{m}\left(R_{\mathrm{E}} / r\right)^{n+2}\left[P_{n}^{m}(\cos \theta) / \sin \theta\right] \cos \phi_{k}^{*} \\
B_{z} & =0
\end{aligned}
$$




\subsection{Equatorial particle trajectories}

Consider first the case for which $(n-m)$ is odd and there exists a class of particle trajectory that is confined to the equatorial plane. If Eqs. 54 and 55 are substituted into Eqs. 17-19, the three differential equations that define the motion of a charged particle in the equatorial plane become

$$
\begin{aligned}
\frac{d^{2} x}{d s^{2}}= & +\left(\frac{Q e}{\gamma m_{0} V}\right)\left[c_{n}^{m}\left(R_{\mathrm{E}} / \varpi\right)^{n+2} \cos \left[m\left(\phi-\phi_{n}^{m}\right)\right]\right. \\
& \left.\times\left[d P_{n}^{m}(\cos \theta) / d \theta\right]_{\theta=\pi / 2}\right] \frac{d y}{d s}, \\
\frac{d^{2} y}{d s^{2}}= & -\left(\frac{Q e}{\gamma m_{0} V}\right)\left[c_{n}^{m}\left(R_{\mathrm{E}} / \varpi\right)^{n+2} \cos \left[m\left(\phi-\phi_{n}^{m}\right)\right]\right. \\
& \left.\times\left[d P_{n}^{m}(\cos \theta) / d \theta\right]_{\theta=\pi / 2}\right] \frac{d x}{d s}, \\
\frac{d^{2} z}{d s^{2}}= & 0 .
\end{aligned}
$$

Equation 61 confirms that the charged particle remains in the equatorial plane $(z=0)$ if $d z / d s=0$, that is if $w=0$.

If Eqs. 59-61 are substituted into Eq. 22, the radius of curvature $(\rho)$ of an equatorial particle trajectory is given by

$$
\begin{aligned}
\frac{1}{\rho^{2}}= & \left(\frac{Q e}{\gamma m_{0} V}\right)^{2}\left[c_{n}^{m}\left(R_{\mathrm{E}} / \varpi\right)^{n+2} \cos \left[m\left(\phi-\phi_{n}^{m}\right)\right]\right. \\
& \left.\times\left[d P_{n}^{m}(\cos \theta) / d \theta\right]_{\theta=\pi / 2}\right]^{2}\left[\left(\frac{d x}{d s}\right)^{2}+\left(\frac{d y}{d s}\right)^{2}\right] .
\end{aligned}
$$

Remembering that $d z / d s=0$ for equatorial trajectories, and using Eq. 21, the expression for the radius of curvature becomes

$$
\begin{aligned}
\rho= & \left(\frac{\gamma m_{0} V}{Q e c_{n}^{m}}\right)\left[\frac{1}{\left|\left[d P_{n}^{m}(\cos \theta) / d \theta\right]_{\theta=\pi / 2}\right|}\right] \\
& \times \frac{\left(\varpi / R_{\mathrm{E}}\right)^{n+2}}{\left|\cos \left[m\left(\phi-\phi_{n}^{m}\right)\right]\right|} .
\end{aligned}
$$

In the limiting case of an axisymmetric, or zonal, magnetic multipole, $m=0, n$ is odd, $c_{n}^{0}=g_{n}^{0}\left(h_{n}^{0} \equiv 0\right)$ and $\left[d P_{n}^{0}(\cos \theta) / d \theta_{\theta=\pi / 2}=-[n(n+1) / 2]^{1 / 2}\right.$, as noted in Sect. 4. If these expressions, together with Eq. 24, are used to simplify Eq. 63, the radius of curvature in the equatorial plane of a zonal $(m=0)$ magnetic multipole becomes

$\rho=\left(\frac{\gamma m_{0} V}{Q e g_{n}^{0}}\right)\left[\frac{\pi^{1 / 2} \Gamma(n / 2+1 / 2)}{2 \Gamma(n / 2+1)}\right]\left(\frac{\varpi}{R_{\mathrm{E}}}\right)^{n+2}$,

since $|\cos (n+1) \pi / 2|=1$ if $n$ is odd. This equation is identical to Eq. 31, and hence Eq. 63 reduces to the correct form in the limiting case of a zonal magnetic multipole $(m=0)$.

For all non-axisymmetric magnetic multipoles, $m>0$ and Eq. 63 can be simplified by using the following relation (Erdélyi et al., 1953; Vol. I, Sect. 3.4, adapted Eq. 23)

$$
\begin{aligned}
\left|\left[d P_{n}^{m}(\cos ) / d \theta\right]_{\theta=\pi / 2}\right|= & 2^{m+1} \pi^{-1 / 2}\left[\frac{2(n-m) !}{(n+m) !}\right]^{1 / 2} \\
& \times \frac{\Gamma(1+n / 2+m / 2)}{\Gamma(1 / 2+n / 2-m / 2)} .
\end{aligned}
$$

This form of the relation is valid for Schmidt's partially normalized associated Legendre function $P_{n}^{m}(\cos \theta)$ in the case for which $m>0,(n-m)$ is odd and hence $|\sin [(n+m) \pi / 2]|=1$. If Eq. 65 is substituted into Eq. 63 , the radius of curvature of a charged particle confined to the equatorial plane of the general tesseral $(0<m<n)$ magnetic multipole becomes

$$
\begin{aligned}
\rho= & \left(\frac{\gamma m_{0} V}{Q e c_{n}^{m}}\right) \llbracket\left[\frac{\pi^{1 / 2}}{2^{m+1}}\left[\frac{(n+m) !}{2(n-m) !}\right]^{1 / 2} \frac{\Gamma(1 / 2+n / 2-m / 2)}{\Gamma(1+n / 2+m / 2)}\right] \\
& \times \frac{\left(\varpi / R_{E}\right)^{n+2}}{\left|\cos \left[m\left(\phi-\phi_{n}^{m}\right)\right]\right|},
\end{aligned}
$$

subject only to the restriction that $(n-m)$ must be odd. It is not possible to derive Eq. 31, which specifies the radius of curvature of a charged-particle trajectory confined to the equatorial plane of an axisymmetric multipole $(m=0)$, simply by putting $m=0$ in Eq. 66, because this latter equation has been derived on the assumption that $m>0$. As noted by Willis and Gardiner (1988), the series expansion for the Schmidt-normalized form of $P_{n}^{m}(\cos \theta)$ yields a value of $P_{n}^{0}(\cos \theta)$ that is too large by a factor $2^{1 / 2}$ in the degenerate case $m=0$; consequently, the value on the right-hand side of Eq. 65 is too large by $2^{1 / 2}$ if $m=0$.

It follows from this last equation that the radius of curvature of a charged particle moving in the equatorial plane $(\theta=\pi / 2)$ of a tesseral $(0<m<n)$ magnetic multipole, for which $(n-m)$ is odd, is directly proportional to $\varpi^{n+2}$ and inversely proportional to $\mid \cos [m(\phi-$ $\left.\left.\phi_{n}^{m}\right)\right] \mid$. Therefore, $\rho \rightarrow \infty$ as $\left|\cos \left[m\left(\phi-\phi_{n}^{m}\right)\right]\right| \rightarrow 0$, which confirms the physically intuitive conclusion that the radius of curvature becomes infinitely large as the particle approaches any one of the $2 m$ neutral lines in the equatorial plane (i.e. $\theta=\pi / 2, \phi=\phi_{n}^{m}+(2 k+1) / 2 m$ for $k=0,1,2, \ldots, 2 m-1)$.

Another particular case of special interest is that of a charged particle moving in the equatorial plane of an anti-symmetric sectorial ( $m=n-1)$ magnetic multipole. The magnetic-field-line configuration in this case has been discussed in detail by Willis and Gardiner (1988). Since $n-m=1$, which is odd, the magnetic field of an anti-symmetric sectorial magnetic multipole is locally perpendicular to the equatorial plane everywhere apart from the $2(n-1)$ equatorial neutral lines defined by $\phi=\phi_{n}^{n-1}+(2 k+1) \pi / 2(n-1)$, where $k=0,1,2, \ldots, 2 n$ - 3. Using the expression $\Gamma(n+1 / 2)=(2 n) ! \pi^{1 / 2} / 2^{2 n} n$ ! (Abramowitz and Stegun, 1972; Chap. 6, Eqs. 6.1 .8 and 6.1.12), Eq. 66 reduces to the following form in the particular case $m=n-1$ 


$$
\begin{aligned}
\rho= & \left(\frac{\gamma m_{0} V}{Q e c_{n}^{n-1}}\right) \llbracket\left[\frac{2^{n} n !}{(2 n) !}\left[\frac{(2 n-1) !}{2}\right]^{1 / 2} \rrbracket\right. \\
& \times \frac{\left(\varpi / R_{\mathrm{E}}\right)^{n+2}}{\left|\cos \left[(n-1)\left(\phi-\phi_{n}^{n-1}\right)\right]\right|} .
\end{aligned}
$$

As in the general case $(0<m<n)$ defined by Eq. 66 , the radius of curvature of a charged particle moving in the equatorial plane of an anti-symmetric sectorial $(m=n-1)$ magnetic multipole is directly proportional to $\varpi^{n+2}$ and inversely proportional to $\mid \cos [(n-1)(\phi-$ $\left.\phi_{n}^{n-1}\right] \mid$. Since $\rho \rightarrow \infty$ as $\mid \cos \left[(n-1)\left(\phi-\phi_{n}^{n-1}\right] \mid \rightarrow 0\right.$, the radius of curvature becomes infinitely large as the particle approaches any one of the $2(n-1)$ neutral lines in the equatorial plane.

In the degenerate case $n=1(m=0)$, which corresponds to a magnetic dipole coincident with the polar axis (Willis and Gardiner, 1988), Eq. 67 reduces to the form

$\rho=\left(\frac{\gamma m_{0} V}{Q e 2^{1 / 2} g_{1}^{0}}\right)\left(\frac{\varpi}{R_{\mathrm{E}}}\right)^{3}$.

This equation is the same as Eq. 32 apart from the introduction of the spurious factor $2^{1 / 2}$. Once again, the discrepancy arises because Eq. 66 is strictly valid only if $m>0$. In the degenerate case $m=0, n=1$, the Schmidt-normalized form of $P_{n}^{m}(\cos \theta)$ that is used in the derivation of Eq. 66 for the case $m>0$ is actually too large by a factor $2^{1 / 2}$, as noted in Sect. 3 of Willis and Gardiner (1988).

\subsection{Meridional particle trajectories}

Consider next the case of particle trajectories that are confined to one of the $2 m$ meridional planes $\phi=\phi_{n}^{m}+$ $(2 k+1) \pi / 2 m=\phi_{k}^{*}$, where $k=0,1,2, \ldots, 2 m-1$. If Eqs. 56-58 are substituted into Eqs. 17-19, the three differential equations that define the motion of a charged particle in one of the $2 m$ meridional planes become

$$
\begin{aligned}
\frac{d^{2} x}{d s^{2}}= & -\left(\frac{Q e}{\gamma m_{0} V}\right)\left[(-1)^{k} m c_{n}^{m}\left(R_{\mathrm{E}} / r\right)^{n+2}\left[P_{n}^{m}(\cos \theta) / \sin \theta\right]\right] \\
& \times \cos \phi_{k}^{*} \frac{d z}{d s}
\end{aligned}
$$

$$
\begin{aligned}
\frac{d^{2} y}{d s^{2}}= & -\left(\frac{Q e}{\gamma m_{0} V}\right)\left[(-1)^{k} m c_{n}^{m}\left(R_{\mathrm{E}} / r\right)^{n+2}\left[P_{n}^{m}(\cos \theta) / \sin \theta\right]\right] \\
& \times \sin \phi_{k}^{*} \frac{d z}{d s},
\end{aligned}
$$

$$
\begin{aligned}
\frac{d^{2} z}{d s^{2}}= & +\left(\frac{Q e}{\gamma m_{0} V}\right)\left[(-1)^{k} m c_{n}^{m}\left(R_{\mathrm{E}} / r\right)^{n+2}\left[P_{n}^{m}(\cos \theta) / \sin \theta\right]\right] \\
& \times\left[\cos \phi_{k}^{*} \frac{d x}{d s}+\sin \phi_{k}^{*} \frac{d y}{d s}\right] .
\end{aligned}
$$

It follows from Eqs. 69 and 70 that

$$
\cos \phi_{k}^{*} \frac{d^{2} y}{d s^{2}}-\sin \phi_{k}^{*} \frac{d^{2} x}{d s^{2}}=0,
$$

which implies that the particle remains in the plane $\phi=\phi_{k}^{*}$ if

$\cos \phi_{k}^{*} \frac{d y}{d s}-\sin \phi_{k}^{*} \frac{d x}{d s}=0$.

If Eqs. 69-71 are substituted into Eq. 22, and Eq. 73 is used to simplify the resulting expression, it follows that $\rho$ is given by

$$
\begin{aligned}
\frac{1}{\rho^{2}}= & \left(\frac{Q e}{\gamma m_{0} V}\right)^{2}\left[(-1)^{k} m c_{n}^{m}\left(R_{\mathrm{E}} / r\right)^{n+2}\left[P_{n}^{m}(\cos \theta) / \sin \theta\right]\right]^{2} \\
& \times\left[\left(\frac{d x}{d s}\right)^{2}+\left(\frac{d y}{d s}\right)^{2}+\left(\frac{d z}{d s}\right)^{2}\right] .
\end{aligned}
$$

Using Eq. 21, the expression for the radius of curvature of a charged particle moving in one of the $2 m$ meridional planes $\phi=\phi_{n}^{m}+(2 k+1) \pi / 2 m=\phi_{k}^{*}$, where $k=0,1,2$, ..., $2 m-1$, becomes

$\rho=\left(\frac{\gamma m_{0} V}{Q e c_{n}^{m}}\right) \frac{\left(r / R_{\mathrm{E}}\right)^{n+2}}{\left|m P_{n}^{m}(\cos \theta) / \sin \theta\right|}$.

It follows from Eq. 7 of Willis and Gardiner (1988) that the relation $m P_{n}^{m}(\cos \theta) / \sin \theta \propto \sin ^{m-1} \theta$ holds for small values of $\sin \theta(\sin \theta<1)$. Therefore, as noted in Sect. 8.1 , the polar axis $(\theta=0, \pi)$ is a neutral line if $m>1$ and in this case Eq. 75 indicates that the radius of curvature becomes infinitely large as the particle approaches this polar neutral line; that is, $\rho \rightarrow \infty$ as $\theta \rightarrow 0$ or $\theta \rightarrow \pi$. Likewise, if $m>0$, Eq. (75) implies that $\rho \rightarrow \infty$ as the particle approaches any one of the $2 m(n-m)$ neutral lines located at the intersections of the $2 m$ meridional planes $\phi=\phi_{n}^{m}+(2 k+1) \pi / 2 m=\phi_{k}^{*}$, where $k=0,1$, $2, \ldots, 2 m-1$, with the $(n-m)$ cones $\theta=\theta_{i}$, where $\theta_{i}$ $(1 \leq i \leq n-m)$ denote the $(n-m)$ roots of the equation $P_{n}^{m}(\cos \theta)=0$ in the range $0<\theta<\pi$. Therefore, the radius of curvature of a planar charged-particle trajectory, confined to one of the $2 m$ meridional planes $\phi=\phi_{k}^{*}$, becomes infinitely large as the particle approaches any one of the $(n-m)$ neutral lines in that particular meridional plane. Moreover, if $(n-m)$ is odd, there are $2 m$ equatorial neutral lines, as noted in Sect. 8.1. In this case, it follows from the results derived in the present section and the previous one that the radius of curvature becomes infinitely large as the particle approaches any equatorial neutral line in either an equatorial or a meridional (planar) trajectory.

In the limiting case of a symmetric sectorial $(m=n)$ magnetic multipole, it follows from Eq. 7 in Willis and Gardiner (1988) that

$\frac{n P_{n}^{n}(\cos \theta)}{\sin \theta}=\frac{[2(2 n) !]^{1 / 2}}{2^{n}(n-1) !} \sin ^{n-1} \theta$.

If Eq. 76 is substituted into Eq. 75, the radius of curvature in the limiting case $m=n$ becomes

$\rho=\left(\frac{\gamma m_{0} V}{Q e c_{n}^{n}}\right)\left[\frac{2^{n}(n-1) !}{[2(2 n) !]^{1 / 2}}\right] \frac{\left(r / R_{\mathrm{E}}\right)^{n+2}}{\sin ^{n-1} \theta}$. 
Since $r^{2}=x^{2}+y^{2}+z^{2}$ and $\sin \theta=\left(x^{2}+y^{2}\right)^{1 / 2} /\left(x^{2}+y^{2}\right.$ $\left.+z^{2}\right)^{1 / 2}$, Eq. 77 is identical to Eq. 48, which confirms that Eq. 75 reduces to the correct form in the special case of a sectorial magnetic multipole $(m=n)$. The physical properties of meridional charged-particle trajectories in sectorial magnetic multipoles are described in Sect. 7 and need not be discussed further here.

In the case of an anti-symmetric sectorial $(m=n-1)$ magnetic multipole, it follows from Eq. 7 in Willis and Gardiner (1988) that

$$
\begin{aligned}
\frac{(n-1) P_{n}^{n-1}(\cos \theta)}{\sin \theta}= & \frac{(n-1)(2 n) !}{2^{n} n !} \\
& \times\left[\frac{2}{(2 n-1) !}\right]^{1 / 2} \sin ^{n-2} \theta \cos \theta .
\end{aligned}
$$

If Eq. 78 is substituted into Eq. 75, the radius of curvature in the case $m=n-1$ becomes

$$
\begin{aligned}
\rho= & \left(\frac{\gamma m_{0} V}{Q e c_{n}^{n-1}}\right) \llbracket \frac{2^{n} n !}{(n-1)(2 n) !}\left[\frac{(2 n-1) !}{2}\right]^{1 / 2} \rrbracket \\
& \times \frac{\left(r / R_{E}\right)^{n+2}}{\sin ^{n-2} \theta|\cos \theta|},
\end{aligned}
$$

which may be rewritten in the form

$$
\begin{aligned}
\rho= & \left(\frac{\gamma m_{0} V}{Q e c_{n}^{n-1}}\right) \llbracket \frac{2^{n} n !}{(n-1)(2 n) !}\left[\frac{(2 n-1) !}{2}\right]^{1 / 2} \rrbracket \\
& \times \frac{\left[\left(x^{2}+y^{2}+z^{2}\right) / R_{\mathrm{E}}^{2}\right]^{(2 n+1) / 2}}{\left[\left(x^{2}+y^{2}\right) / R_{\mathrm{E}}^{2}\right]^{(n-2) / 2}\left(|z| / R_{\mathrm{E}}\right)}
\end{aligned}
$$

since $r^{2}=x^{2}+y^{2}+z^{2}, \sin \theta=\left(x^{2}+y^{2}\right)^{1 / 2} /\left(x^{2}+y^{2}+\right.$ $\left.z^{2}\right)^{1 / 2}$ and $\cos \theta=z /\left(x^{2}+y^{2}+z^{2}\right)^{1 / 2}$. The physical interpretation of Eq. 80, which applies to an antisymmetric sectorial $(m=n-1)$ magnetic multipole, is entirely analogous to the interpretation of Eq. 75, which applies to a tesseral $(0<m<n)$ magnetic multipole. In the particular case $m=n-1$, for which $(n-m)$ is clearly odd, there is only one root of the equation $P_{n}^{n-1}(\cos \theta)=0$ in the range $0<\theta<\pi$, namely $\theta=\pi / 2$. Therefore, apart from the neutral line coincident with the polar axis if $n>2$, there are only the $2(n-1)$ neutral lines in the equatorial plane.

As already noted for equatorial trajectories, the degenerate case $n=1(m=0)$ corresponds to a magnetic dipole coincident with the polar axis. Equation 80 is inapplicable in the degenerate case $m=0$ because the derivation of this expression for the radius of curvature is valid only if $m>0$. In the particular case $n=2(m=1)$, which corresponds to a "normal" (or planar) quadrupole aligned with the polar axis, it follows from Eq. 80 that $\rho \propto$ $r^{5}|z|^{-1}$, where $z$ denotes perpendicular distance from the equatorial neutral line in either of the two meridional planes $\phi=\phi_{2}^{1}+\pi / 2$ and $\phi=\phi_{2}^{1}+3 \pi / 2(k=0,1)$. In addition, for equatorial trajectories in the particular case $n=2(m=1)$, it follows from Eq. 67 that $\rho \propto \varpi^{5}|\xi|^{-1}$, where $\xi$ denotes equatorial distance perpendicular to either of these (equatorial) neutral lines.

Similarly, if $m=n=2$ it follows from Eq. (48) that $\rho \propto r^{5} \varpi^{-1}$ where $\varpi$ again denotes perpendicular distance from the polar axis $(\theta=0, \pi)$ in one of the following four meridional planes $\phi=\phi_{2}^{2}+\pi / 4, \phi$ $=\phi_{2}^{2}+3 \pi / 4, \phi=\phi_{2}^{2}+5 \pi / 4$ and $\phi=\phi_{2}^{2}+7 \pi / 4$ $(k=0,1,2,3)$. The essential equivalence of the functional form of the two quadrupolar radii of curvature ( $m=1, n=2$ and $m=n=2$ ) arises because the corresponding magnetic fields differ only in terms of their different orientations with respect to the system of spherical polar coordinates (Willis and Gardiner, 1988). This result is an extension to the case $n=2$ of the essential equivalence of the functional form of the two dipolar radii of curvature $(m=0, n=1$ and $m=n=1)$, as noted in the discussion following immediately after Eq. 49. By analogy with the corresponding conclusion for the configurations of multipole magnetic fields (Willis and Gardiner, 1988), this result cannot be extended to the radii of curvature of planar particle trajectories in higher-degree magnetic multipoles $(n>2)$.

\section{Illustrative charged-particle trajectories}

The purpose of this section is to provide a few illustrative examples of planar charged-particle trajectories in low-degree $(n \leq 3)$ multipole magnetic fields. All trajectories presented in the following figures have been derived by stepwise numerical integration of the exact equations of motion of a charged particle in a static magnetic field. In each example, distances are expressed in terms of the radius of the Earth $\left(R_{\mathrm{E}}\right)$ as unit distance. Since the particle trajectories are merely intended to be illustrative, the strength of each lowdegree multipole is arbitrarily assumed to be defined by the appropriate spherical harmonic coefficients in the International Geomagnetic Reference Field (IGRF) for 1990 (Langel, 1992). The energy of the particle (proton) is then adjusted to give trajectories that lie within a sphere of radius $4 R_{\mathrm{E}}$ yet still illustrate the basic principles of particle motion in low-degree multipole magnetic fields. Although the various aspects of numerical accuracy are not discussed in any detail, considerable care has been taken to ensure that the numerical integrations are accurate. This accuracy has been achieved by checking all results using different standard numerical integration routines (e.g. Adams, Backward Differentiation Formulae and Runge-KuttaMerson) and different numerical tolerances (e.g. $10^{-4}$ to $10^{-14}$ ) in these integration routines.

Figure 1 shows an illustrative trajectory for a $1-\mathrm{GeV}$ proton confined to the equatorial plane of a zonal magnetic dipole $\left(g_{1}^{0}=-29775 \mathrm{nT}\right)$, obtained by stepwise numerical integration of Eqs. 27-29 in the case $n=1$. This trajectory illustrates the classic result, derived by Störmer (1955), that the radius of curvature $(\rho)$ of a charged particle moving in the equatorial plane of a magnetic dipole is proportional to the cube of the equatorial distance $(\varpi)$ of the particle from the dipole, 


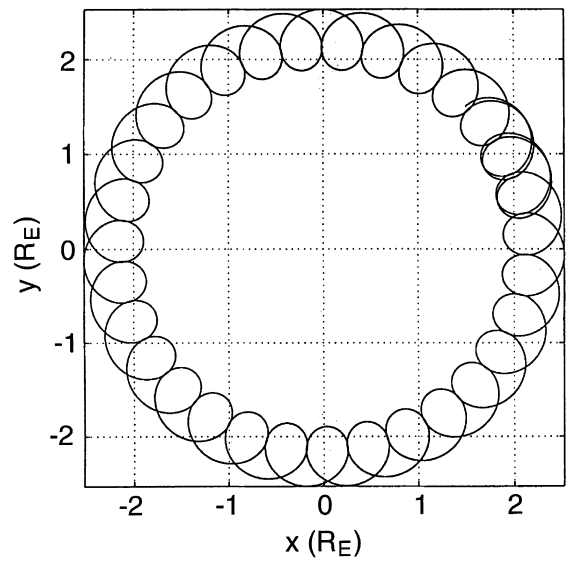

Fig. 1. Planar equatorial trajectory of a proton $(1 \mathrm{GeV})$ in a zonal dipole magnetic field $\left(m=0, n=1 ; g_{1}^{0}=-29775 \mathrm{nT}\right)$

namely $\rho \propto \varpi^{3}$ (cf. Sect. 6). Similarly, Fig. 2 shows an illustrative trajectory for a $300-\mathrm{keV}$ proton confined to the equatorial plane of a zonal magnetic octupole $\left(g_{3}^{0}=1315 \mathrm{nT}\right)$, obtained by stepwise numerical integration of Eqs. 27-29 in the case $n=3$. The radius of curvature of this trajectory varies with equatorial distance from the octupole according to the relation $\rho \propto \pi^{5}$.

Figure 3 shows a pair of sample trajectories for a 500$\mathrm{keV}$ proton confined to a supplementary pair of the four special meridional planes $\phi=\phi_{2}^{2}+(2 k+1) \pi / 4$ $(k=0,1,2,3)$ of a symmetric sectorial magnetic quadrupole $\left(g_{2}^{2}=1693 \mathrm{nT}, h_{2}^{2}=-380 \mathrm{nT}\right)$, obtained by stepwise numerical integration of Eqs. $42-44$ in the case $m=n=2$ (cf. Sect. 7). It follows from Eq. 48 that the radius of curvature of every such planar trajectory varies according to the relation $\rho \propto r^{5} \varpi^{-1}$. Since $n=2$ (i.e. $n>1$ ), the polar axis is a magnetic neutral line (cf. Sect. 7) and the relation $\rho \propto r^{5} \varpi^{-1}$ implies that $\rho \rightarrow \infty$ as $\varpi \rightarrow 0$. The trajectories shown in Fig. 3 confirm the physically intuitive conclusion that the radius of curvature becomes infinitely large as the particle approaches the polar magnetic neutral line (i.e. the $z$-axis).

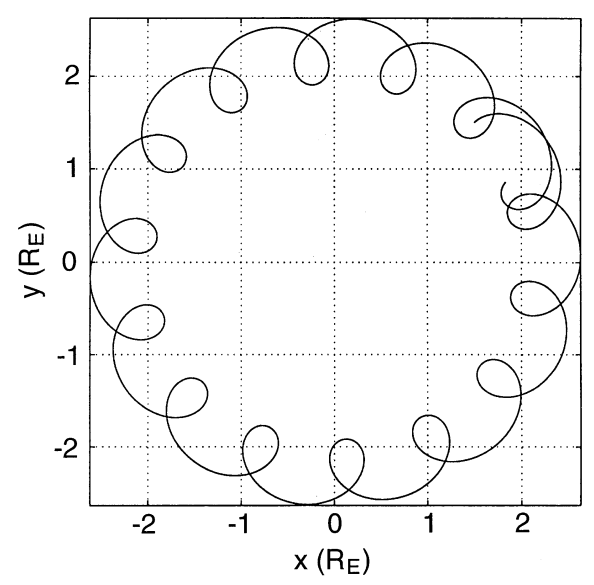

Fig. 2. Planar equatorial trajectory of a proton $(300 \mathrm{keV})$ in a zonal octupole magnetic field $\left(m=0, n=3 ; g_{3}^{0}=1315 \mathrm{nT}\right)$

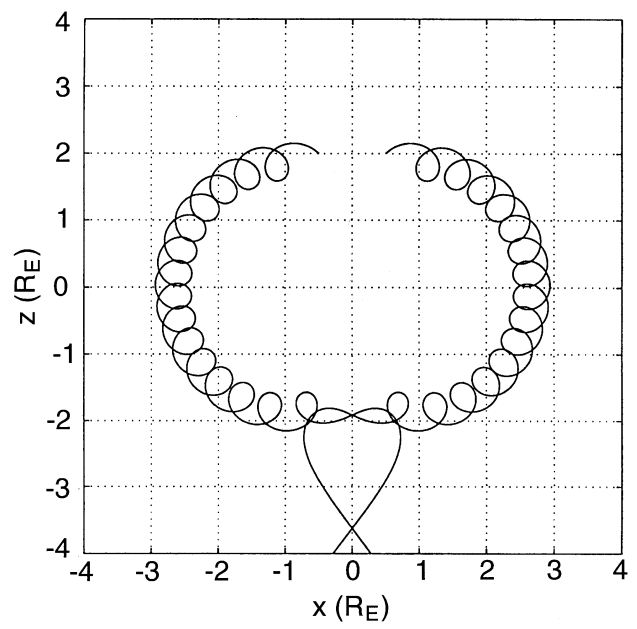

Fig. 3. Planar meridional trajectories of a proton $(500 \mathrm{keV})$ in a symmetric sectorial quadrupole magnetic field $(m=2, n=2$; $\left.g_{2}^{2}=1693 \mathrm{nT}, h_{2}^{2}=-380 \mathrm{nT}\right)$

It only remains to illustrate the various particle trajectories in tesseral multipole magnetic fields (cf. Sect. 8). Figure 4 shows both an equatorial and a meridional trajectory for a proton in an anti-symmetric sectorial magnetic quadrupole $\left(g_{2}^{1}=3058 \mathrm{nT}\right.$, $\left.h_{2}^{1}=-2278 \mathrm{nT}\right)$, for which $(n-m)$ is odd. The equatorial trajectory shown in Fig. 4 is for a $3-\mathrm{MeV}$ proton and is obtained by stepwise numerical integration of Eqs. 59-61 in the case $m=1, n=2$ (cf. Sect. 8.2). The meridional trajectory is for a $1-\mathrm{MeV}$ proton and is obtained by stepwise numerical integration of Eqs. $69-71$ in the case $m=1, n=2$ (cf. Sect. 8.3). It follows from Eq. 67 that the radius of curvature $(\rho)$ of a planar charged-particle trajectory confined to the equatorial plane of this anti-symmetric sectorial magnetic multipole is directly proportional to $\varpi^{4}$ and inversely proportional to $\cos \left(\phi-\phi_{2}^{1}\right)$. Since $\rho \rightarrow \infty$ as $\cos \left(\phi-\phi_{2}^{1}\right) \rightarrow 0$, the radius of curvature becomes infinitely large as the particle approaches either of the two magnetic neutral lines in the equatorial plane, which are defined by $\phi=\phi_{2}^{1}+(2 k+1) \pi / 2(k=0,1)$. Like-

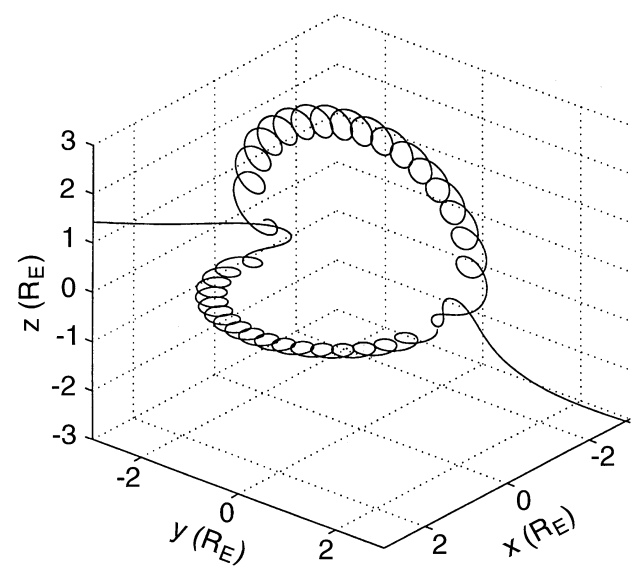

Fig. 4. Planar equatorial $(3 \mathrm{MeV})$ and meridional $(1 \mathrm{MeV})$ trajectories of a proton in an anti-symmetric sectorial quadrupole magnetic field ( $\left.m=1, n=2 ; g_{2}^{1}=3058 \mathrm{nT}, h_{2}^{1}=-2278 \mathrm{nT}\right)$ 
wise, it follows from Eq. 75 that the radius of curvature of a charged-particle trajectory confined to the supplementary pair of meridional planes passing through these two magnetic neutral lines becomes infinitely large as the particle approaches either one of these neutral lines. The equatorial and meridional trajectories shown in Fig. 4 confirm these conclusions.

Similarly, Fig. 5 shows an equatorial and two meridional trajectories for a proton in an antisymmetric sectorial magnetic octupole $\left(g_{3}^{2}=1246 \mathrm{nT}\right.$, $h_{3}^{2}=293 \mathrm{nT}$ ). The equatorial trajectory shown in Fig. 5 is for a $50-\mathrm{keV}$ proton and the meridional trajectories are for a $5-\mathrm{keV}$ proton. This figure confirms that the radius of curvature of any particle trajectory becomes infinite as the particle approaches the polar magnetic neutral line (i.e. the $z$-axis) or either of the two (supplementary) equatorial magnetic neutral lines (i.e. the $x$ - and $y$-axes).

Consider next particle trajectories in tesseral multipole magnetic fields for which $(n-m)$ is even rather than odd. Figure 6 shows two illustrative meridional trajectories for a $100-\mathrm{keV}$ proton in a tesseral magnetic octupole $\left(g_{3}^{1}=-2240 \mathrm{nT}, h_{3}^{1}=-287 \mathrm{nT}\right)$. The magnetic field of this multipole is locally perpendicular to the two meridional planes $\phi=\phi_{3}^{1}+(2 k+1) \pi / 2(k=0,1)$ everywhere apart from the four neutral lines defined by the intersections of these two meridional planes with the two cones $\theta=\theta_{1}$ and $\theta=\theta_{2}$, where $\theta_{1}$ and $\theta_{2}$ denote the two roots of the equation $P_{3}^{1}(\cos \theta)=0$ in the range $0<$ $\theta<\pi$ (cf. Sect. 8.1). It follows from Willis and Young (1987) that $\theta_{1}=\arccos (1 / \sqrt{ } 5)=63^{\circ} 26^{\prime} 06^{\prime \prime}$ and $\theta_{2}=\pi$ $-\arccos (1 / \sqrt{ } 5)=116^{\circ} 33^{\prime} 54^{\prime \prime}$ (after correcting for the typographical omission of the solidus sign in the earlier paper). Figure 6 confirms that the radius of curvature of a planar charged-particle trajectory, confined to the supplementary pair of meridional planes, becomes infinitely large as the particle approaches any one of the four neutral lines defined by the intersection of these planes with the cones $\theta=\theta_{1}$ and $\theta=\theta_{2}$.

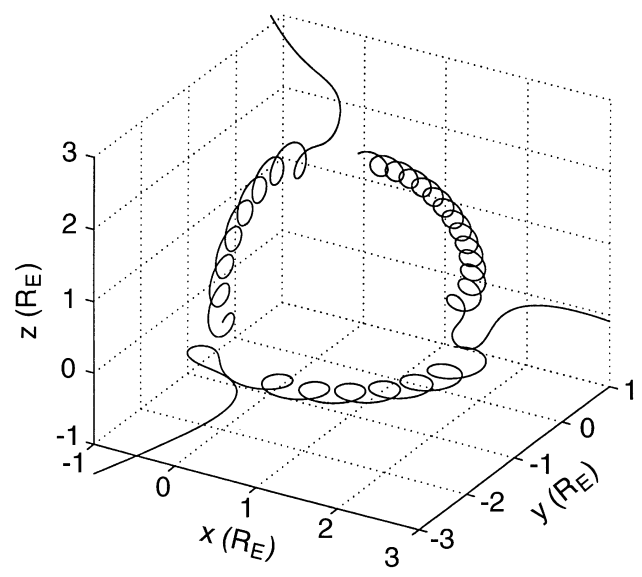

Fig. 5. Planar equatorial $(50 \mathrm{keV})$ and meridional $(5 \mathrm{keV})$ trajectories of a proton in an anti-symmetric sectorial octupole magnetic field $\left(m=2, n=3 ; g_{3}^{2}=1246 \mathrm{nT}, h_{3}^{2}=293 \mathrm{nT}\right)$

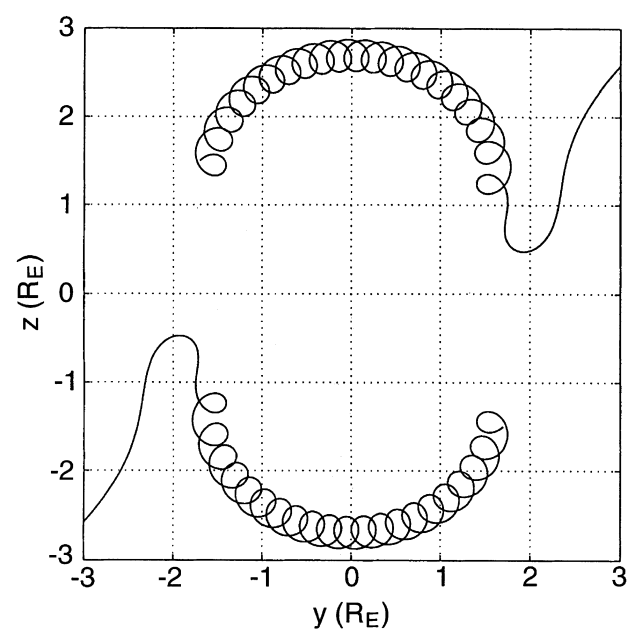

Fig. 6. Planar meridional trajectories of a proton $(100 \mathrm{keV})$ in a tesseral octupole magnetic field ( $m=1, n=3 ; g_{3}^{1}=-2240 \mathrm{nT}, h_{3}^{1}=$ $-287 \mathrm{nT})$

\section{Scattering of charged particles at a magnetic neutral line}

Figures 3-6 illustrate the "non-adiabatic" scattering of charged particles near a magnetic neutral line, in the sense that Alfvén's "guiding-centre" approximation (Alfvén and Fälthammar, 1963; Northrop, 1963) is violated in the immediate vicinity of each neutral line. In the static magnetic fields considered here, the "guidingcentre" approximation is valid if the particle's radius of gyration is small compared with the characteristic scale length of spatial variations in the magnetic field (Chapman, 1961). Since it has been shown in this paper that the radius of curvature of a particle's trajectory (i.e. the instantaneous radius of gyration) becomes infinitely large as the particle approaches a magnetic neutral line, the "guiding-centre" approximation is not valid in the immediate vicinity of such a neutral line.

Although a detailed discussion of the dynamics of charged particles in multipole magnetic fields is beyond the intended scope of the present paper, a few brief comments should be made about the two distinct classes of planar charged-particle trajectory. For an axisymmetric magnetic multipole $(m=0)$ of odd degree ( $n$ odd), there exists a class of equatorial trajectory that can clearly never intersect a neutral line (cf. Sect. 6, also Figs. 1 and 2). In the case of a dipole $(n=1)$, it is known that the general (three-dimensional) equations of motion for a trapped particle cannot be solved analytically (Dragt and Finn, 1976), whereas the equations of motion for a particle confined to the equatorial plane can be solved in terms of elliptic functions (Störmer, 1955; Avrett, 1962; Dragt, 1965). A similar statement holds for charged-particle motion in the equatorial plane of an axisymmetric magnetic multipole of arbitrary degree $n$, provided that $n$ is odd. Therefore, every equatorial trajectory in an axisymmetric magnetic multipole of arbitrary (odd) degree is regular (integrable) and conserves an adiabatic invariant (such as $\mu$ ). 
This class of planar trajectory is a special case that is dynamically distinct from the rest of phase space.

Conversely, for a non-axisymmetric (sectorial or tesseral) magnetic multipole $(m \neq 0)$, every equatorial or meridional trajectory eventually intersects a neutral line (cf. Sects. 7 and 8, also Figs. 3-6), apart from the degenerate case $m=n=1$, which corresponds to a dipole lying in the equatorial plane (cf. Sect. 7). It follows from the analytic results presented in Sects. 7 and 8 , as well as from the form of the computed trajectories presented in Figs. 3-6, that a particle behaves irregularly when it intersects a neutral line. Therefore, every equatorial or meridional trajectory in a non-axisymmetric magnetic multipole $(n>1)$ is irregular (non integrable) and does not conserve a single invariant. This class of planar trajectory may well be dynamically indistinct from the rest of phase space, although a completely rigorous resolution of this matter must await a more detailed investigation.

Notwithstanding the need for a more detailed investigation of the dynamics of charged particles in multipole magnetic fields, it should be noted that the results outlined are analogous to those that apply to charged-particle motion in magnetic reversals (e.g. Büchner and Zelenyi, 1986, 1989; Chen 1992; Chapman, 1993, 1994; Chapman and Watkins, 1993, 1995). In magnetic reversals with parabolic field lines, the equations of particle motion are only integrable in the special "equatorial" plane $(z=0)$, which is the only plane that is everywhere perpendicular to the parabolic field lines. Trajectories confined to this special plane (i.e. $z=0, d z / d t=0)$ are regular and conserve an exact invariant, whereas trajectories not confined to this plane are irregular (chaotic) and do not conserve a single invariant. In general, chaotic, or stochastic, trajectories (or sections of such trajectories) access a much more extensive region of phase space than regular trajectories, which are constrained in phase space by the existence of adiabatic invariants.

\section{Conclusions}

In his book The Polar Aurora, Störmer (1955) obtained an exact equation for the radius of curvature of the trajectory of a charged particle whose orbital motion is confined to the equatorial plane of a magnetic dipole. Every equatorial trajectory has the remarkable geometrical property that its radius of curvature $(\rho)$ at any point is proportional to the cube of its (equatorial) distance $(\varpi)$ from the magnetic dipole, that is $\rho \propto \varpi^{3}$. This paper extends Störmer's result by deriving equally remarkable exact equations for the radii of curvature of all possible planar charged-particle trajectories in an individual static magnetic multipole of arbitrary degree $(n)$ and order $(m)$.

In general, planar charged-particle trajectories arise wherever there exists a plane (or planes) such that the multipole magnetic field is everywhere perpendicular to this plane (or planes), apart from possibly at a set of neutral lines where the magnetic field actually vanishes.
For example, the magnetic field of an axisymmetric $(m=0)$, or zonal, magnetic multipole is everywhere perpendicular to the equatorial plane, provided the degree $(n)$ of the multipole is odd. In this general axisymmetric case, the radius of curvature of the equatorial particle trajectory is defined by the relation $\rho \propto \varpi^{n+2}$ ( $n$ odd), which reduces to $\rho \propto \varpi^{3}$ if $n=1$ (cf. Eqs. 31 and 32). Therefore, the general result for the radius of curvature of the trajectory of a charged particle confined to the equatorial plane of a static axisymmetric magnetic multipole reduces to Störmer's classic result in the limiting case of a magnetic dipole $(n=1)$. There are no planar charged-particle trajectories in the case of an axisymmetric magnetic multipole of even degree (i.e. $n$ even).

In the case of a general symmetric sectorial $(m=n)$ magnetic multipole, the magnetic field is locally perpendicular to $2 n$ meridional planes everywhere apart from the polar axis $(\theta=0, \pi)$, which is a magnetic neutral line if $n>1$. The radius of curvature of a charged particle confined to any one of these $2 n$ meridional planes varies according to the relation $\rho \propto r^{2 n+1} / \varpi^{n-1}$, where $r$ denotes radial distance from the symmetric sectorial multipole and $\varpi$ denotes perpendicular distance from the polar axis (cf. Eq. 48). Therefore, the radius of curvature becomes infinitely large as the particle approaches the polar magnetic neutral line $(n>1)$, as is intuitively clear on physical grounds.

In the case of a general tesseral $(0<m<n)$ magnetic multipole, the magnetic field is locally perpendicular to $2 m$ meridional planes everywhere apart from the polar axis, which is a neutral line if $m>1$, and the $2 m(n-m)$ neutral lines lying at the intersections of the $2 \mathrm{~m}$ meridional planes with the $(n-m)$ cones $\theta=\theta_{i}$, where $\theta_{i}(1 \leq i \leq n-m)$ denote the $(n-m)$ different roots of the equation $P_{n}^{m}(\cos \theta)=0$ in the range $0<\theta<\pi$. Thus there are $2 m(n-m)$ neutral lines apart from the polar axis in the case of the general tesseral magnetic multipole. Moreover, if $(n-m)$ is odd, one of the $(n-m)$ cones coincides with the magnetic equator $(\theta=\pi / 2)$ and in this case the magnetic field is perpendicular to the equatorial plane everywhere apart from the $2 m$ equatorial neutral lines. Therefore, planar trajectories exist in $2 m$ meridional planes and the equatorial plane if $(n-m)$ is odd, but exist only in $2 m$ meridional planes if $(n-m)$ is even.

If $(n-m)$ is odd, the radius of curvature of an equatorial particle trajectory is directly proportional to $\varpi^{n+2}$, where $\varpi$ denotes equatorial radial distance from the multipole, and inversely proportional to $\cos [m$ $\left.\left(\phi-\phi_{n}^{m}\right)\right]$ (cf. Eq. 66). Since $\cos \left[m\left(\phi-\phi_{n}^{m}\right)\right]$ vanishes at the $2 m$ equatorial neutral lines, the radius of curvature becomes infinitely large as the particle approaches any one of these neutral lines. This result holds for an anti-symmetric sectorial $(m=n-1)$ multipole because $n-m=1$, which is odd (cf. Eq. 67).

The radius of curvature of a meridional particle trajectory is directly proportional to $r^{n+2}$, where $r$ denotes radial distance from the multipole, and inversely proportional to $P_{n}^{m}(\cos \theta) / \sin \theta \quad(\mathrm{cf}$. Eq. 75). Since $P_{n}^{m}(\cos \theta) / \sin \theta \propto \sin ^{m-1} \theta$ for small values of $\theta$ (Willis 
and Gardiner, 1988) and hence the polar axis $(\theta=0, \pi)$ is a neutral line if $m>1$, the radius of curvature becomes infinitely large as the particle approaches the polar magnetic neutral line. Likewise, the radius of curvature becomes infinitely large as the particle approaches any one of the $2 m(n-m)$ neutral lines located at the intersections of the $2 m$ meridional planes with the $(n-m)$ cones defined by the roots of the equation $P_{n}^{m}(\cos \theta)=0(0<\theta<\pi)$

Planar charged-particle trajectories, which illustrate these theoretical results, are presented in Sect. 9 for lowdegree $(n \leq 3)$ magnetic multipoles. These illustrative planar trajectories are computed by stepwise numerical integration of the exact equations of particle motion. The computed planar trajectories clearly show the "nonadiabatic" scattering of charged particles at equatorial, meridional and polar neutral lines in the case of nonaxisymmetric magnetic multipoles $(m \neq 0)$. It should be emphasized, however, that all the theoretical and computational results presented in this paper refer to planar charged-particle trajectories in an individual static magnetic multipole field.

This non-adiabatic scattering of charged particles casts some doubt on the possible existence of belts of stably trapped radiation in any non-axisymmetric multipole magnetic field that might exist during a geomagnetic polarity reversal. There could still be classes of complicated, three-dimensional trajectories, corresponding to the confinement ("trapping") of charged particles in non-axisymmetric multipole magnetic fields, but this possibility requires further investigation. Similarly, further research is required on the different regions of phase space defined by regular (integrable) and irregular (non integrable) trajectories.

Acknowledgements. The authors thank Mr S. R. Crothers and Mrs J. Foster for considerable help with the preparation of the paper. Part of the research reported was undertaken while VJB pursued a twelve-month period of Industrial Training at the Rutherford Appleton Laboratory as part of the BSc Honours Degree Course in Mathematics with Computing at the University of Portsmouth. The paper was completed while ARG was funded by the UK Engineering and Physical Sciences Research Council under grants GR/F85659 and GR/H61445.

Topical Editor K.-H. Glaßmeier thanks S. C. Chapman and A. Brekke for their help in evaluating this paper.

\section{References}

Abramowitz, M., and I. A. Stegun, Handbook of Mathematical Functions, Dover Publications, New York, 1972.

Akasofu, S.-I., and S. Chapman, Solar-Terrestrial Physics, Oxford University Press, London, 1972.

Alfvén, H., and C.-G. Fälthammar, Cosmical Electrodynamics, 2nd Ed., Oxford University Press, London, 1963.

Avrett, E. H., Particle motion in the equatorial plane of a dipole magnetic field, J. Geophys. Res., 67, 53-58, 1962.

Backus, G. E., The field lines of an axisymmetric magnetic field, Geophys. J., 93, 413-417, 1988.

Bogue, S. W., and K. A. Hoffman, Morphology of geomagnetic reversals, Rev. Geophys., 25, 910-916, 1987.

Bogue, S. W., and R. T. Merrill, The character of the field during geomagnetic reversals, Annu. Rev. Earth Planet. Sci., 20, 181219, 1992.
Büchner, J., and L. M. Zelenyi, Deterministic chaos in the dynamics of charged particles near a magnetic field reversal, Phys. Lett. A, 118, 395-399, 1986.

Büchner, J., and L. M. Zelenyi, Regular and chaotic charged particle motion in magnetotaillike field reversals: 1 . Basic theory of trapped motion, J. Geophys. Res., 94, 11821-11842, 1989.

Chapman, S., Scale times and scale lengths of variables: with geomagnetic and ionospheric illustrations, Proc. Phys. Soc., 77, $424-432,1961$.

Chapman, S., and J. Bartels, Geomagnetism, Volumes I and II, Oxford University Press, London, 1940.

Chapman, S. C., Chaotic single-particle dynamics in a multitimescale parameterizable field reversal, Ann Geophysicae, 11, 239-247, 1993.

Chapman, S. C., Properties of single-particle dynamics in a parabolic magnetic reversal with general time dependence, J. Geophys. Res., 99, 5977-5985, 1994.

Chapman, S. C., and N. W. Watkins, Parameterization of chaotic particle dynamics in a simple time-dependent field reversal, J. Geophys. Res., 98, 165-177, 1993.

Chapman, S. C., and N.W. Watkins, Delay coordinates: a sensitive indicator of nonlinear dynamics in single-charged-particle motion in magnetic reversals, Ann. Geophysicae, 13, 836-842, 1995.

Chauvin, A., P. Roperch, and R. A. Duncan, Records of geomagnetic reversals from volcanic islands of French Polynesia 2. Paleomagnetic study of a flow sequence (1.2-0.6 Ma) from the island of Tahiti and discussion of reversal models, J. Geophys. Res., 95, 2727-2752, 1990.

Chen, J., Nonlinear dynamics of charged particles in the magnetotail, J. Geophys. Res., 97, 15011-15050, 1992.

Clement, B. M., Geographical distribution of transitional VGPs: evidence for non-zonal equatorial symmetry during the Matuyama-Brunhes geomagnetic reversal, Earth Planet. Sci. Lett., 104, 48-58, 1991.

Clement, B. M., and D. V. Kent, A detailed record of the lower Jaramillo polarity transition from a southern hemisphere, deepsea sediment core, J. Geophys. Res., 89, 1049-1058, 1984a.

Clement, B. M., and D. V. Kent, Latitudinal dependency of geomagnetic polarity transition durations, Nature, 310, 488491, 1984b.

Clement, B. M., and D. V. Kent, A comparison of two sequential geomagnetic polarity transitions (upper Olduvai and lower Jaramillo) from the Southern Hemisphere, Phys. Earth Planet. Int., 39, 301-313, 1985.

Clement, B. M., and D. V. Kent, A southern hemisphere record of the Matuyama-Brunhes polarity reversal, Geophys. Res. Lett., 18, 81-84, 1991.

Coe, R. S., M. Prévot, and P. Camps, New evidence for extraordinarily rapid change of the geomagnetic field during a reversal, Nature, 374, 687-692, 1995.

Dodson, R., J. R. Dunn, M. Fuller, I. Williams, H. Ito, V.A. Schmidt, and Y.-M. Wu, Palaeomagnetic record of a late Tertiary field reversal, Geophys. J. R. Astron. Soc., 53, 373-412, 1978.

Dragt, A. J., Trapped orbits in a magnetic dipole field, Rev. Geophys., 3, 255-298, 1965.

Dragt, A. J., and J. M. Finn, Insolubility of trapped particle motion in a magnetic dipole field, J. Geophys. Res., 81, 2327-2340, 1976.

Erdélyi A., W. Magnus, F. Oberhettinger, and F. G. Tricomi, Higher Transcendental Functions, Volume I, McGraw-Hill, New York, 1953.

Fuller, M., I. Williams, and K. A. Hoffman, Paleomagnetic records of geomagnetic field reversals and the morphology of the transitional fields, Rev. Geophys. Space Phys., 17, 179-203, 1979.

Herrero-Bervera, E., and F. Theyer, Non-axisymmetric behaviour of Olduvai and Jaramillo polarity transitions recorded in north-central Pacific deep-sea sediments, Nature, 322, 159-162, 1986. 
Hess, W. N., The Radiation Belt and Magnetosphere, Blaisdell, Waltham, Mass., 1968.

Hide, R., Self-exciting dynamos and geomagnetic polarity changes, Nature, 293, 728-729, 1981.

Hide, R., On the role of rotation in the generation of magnetic fields by fluid motions, Phil. Trans. Roy. Soc., London, A306, 223-234, 1982.

Hoffman, K. A., Polarity transition records and the geomagnetic dynamo, Science, 196, 1329-1332, 1977.

Hoffman, K. A., Behaviour of the geodynamo during reversal: a phenomenological model, Earth Planet. Sci. Lett., 44, 7-17, 1979.

Hoffman, K. A., Quantitative description of the geomagnetic field during the Matuyama-Brunhes polarity transition, Phys. Earth Planet. Int., 24, 229-235, 1981.

Hoffman, K. A., The testing of geomagnetic reversal models: recent developments, Phil. Trans. R. Soc., London, A306, 147-159, 1982.

Hoffman, K. A., Geomagnetic reversals and excursions: their paleomagnetic record and implications for the geodynamo, Rev. Geophys. Space Phys., 21, 614-620, 1983.

Hoffman, K. A., Transitional behavior of the geomagnetic field, J. Geomagn. Geoelectr, 37, 139-146, 1985.

Hoffman, K. A., Transitional field behaviour from Southern Hemisphere lavas: evidence for two-stage reversals of the geodynamo, Nature, 320, 228-232, 1986.

Hoffman, K. A., and M. Fuller, Transitional field configurations and geomagnetic reversal, Nature, 273, 715-718, 1978.

Jacobs, J. A., Reversals of the Earth's Magnetic Field, Adam Hilger, Bristol, 1984.

Jeffreys, B., Derivations of the equation for the field lines of an axisymmetric multipole, Geophys. J., 92, 355-356, 1988.

Laj, C., S. Guitton, C. Kissel, and A. Mazaud, Complex behaviour of the geomagnetic field during three successive polarity reversals, 11-12 m.y. B.P., J. Geophys. Res., 93, 11655-11666, 1988.

Langel, R. A., International Geomagnetic Reference Field: the sixth generation, J. Geomagn. Geoelectr., 44, 679-707, 1992.

Mankinen, E. A., M. Prévot, C. S. Grommé, and R. S. Coe, The Steens Mountain (Oregon) geomagnetic polarity transition 1. Directional history, duration of episodes, and rock magnetism, J. Geophys. Res., 90, 10393-10416, 1985.

McFadden, P. L., and R. T. Merrill, History of Earth's magnetic field and possible connections to core-mantle boundary processes, J. Geophys. Res., 100, 307-316, 1995.

Merrill, R. T., and M. W. McElhinny, The Earth's Magnetic Field: Its History, Origin and Planetary Perspective, Academic Press, London, 1983.

Northrop, T. G., The Adiabatic Motion of Charged Particles, WileyInterscience, New York, 1963.

Prévot, M., E. A. Mankinen, C. S. Grommé, and R. S. Coe, How the geomagnetic field vector reverses polarity, Nature, 316, 230 234, 1985a.

Prévot, M., E. A. Mankinen, R. S. Coe, and C. S. Grommé, The Steens Mountain (Oregon) geomagnetic polarity transition 2. Field intensity variations and discussion of reversal models, J. Geophys. Res., 90, 10417-10448, 1985b.

Rishbeth, H., The quadrupole ionosphere, Ann. Geophysicae, 3, 293-298, 1985.

Roederer, J. G., Dynamics of Geomagnetically Trapped Radiation, Springer-Verlag, Berlin, Heidelberg, New York, 1970.

Roederer, J. G., Geomagnetic field distortions and their effects on radiation belt particles, Rev. Geophys. Space Phys., 10, 599-630, 1972.

Roperch, P., and A. Chauvin, Transitional geomagnetic field behavior: volcanic records from French Polynesia, Geophys. Res. Lett., 14, 151-154, 1987.
Roperch, P., and R. A. Duncan, Records of geomagnetic reversals from volcanic islands of French Polynesia 1. Paleomagnetic study of a polarity transition in a lava sequence from the island of Huahine, J. Geophys. Res., 95, 2713-2726, 1990.

Sandström, A. E., Cosmic Ray Physics, North-Holland, Amsterdam, 1965.

Siscoe, G. L., Solar-terrestrial relations: Stone age to space age, Technol. Rev., 78 (3), 26-37, 1976a.

Siscoe, G. L., Minimum-effect model of geomagnetic excursions applied to auroral zone locations, J. Geomagn. Geoelectr., 28, 427-436, 1976b.

Siscoe, G. L., Long-term aspects of magnetospheric variability, in Physics of Solar Planetary Environments, Vol. II, Ed. D.J. Williams, AGU, Washington D.C., pp 973-1004, 1976c.

Siscoe, G. L., Towards a comparative theory of magnetospheres, in Solar System Plasma Physics, Vol. II (Magnetospheres), Eds. C.F. Kennel, L.J. Lanzerotti, and E.N. Parker, North-Holland Amsterdam, pp 319-402, 1979.

Siscoe, G. L., and C.-K. Chen, The paleomagnetosphere, J. Geophys. Res., 80, 4675-4680, 1975.

Siscoe, G. L., and L. Christopher, Effects of geomagnetic dipole variations on the auroral zone locations, J. Geomagn. Geoelectr, 27, 485-489, 1975.

Siscoe, G. L., and N. U. Crooker, Auroral zones in a quadrupole magnetosphere, J. Geomagn. Geoelectr., 28, 1-9, 1976.

Siscoe, G. L., and D. G. Sibeck, Effects of nondipole components on auroral zone configurations during weak dipole field epochs, J. Geophys. Res., 85, 3549-3556, 1980.

Siscoe, G. L., C.-K. Chen, and M. Harel, On the altitude of the magnetopause during geomagnetic reversals, J. Atmos. Terr. Phys., 38, 1327-1331, 1976.

Stern, D. P., Representation of magnetic fields in space, Rev. Geophys. Space Phys., 14, 199-214, 1976.

Störmer, C., The Polar Aurora, Oxford University Press, London, 1955.

Theyer, F., E. Herrero-Bervera, V. Hsu, and S. R. Hammond, The zonal harmonic model of polarity transitions: a test using successive reversals, J. Geophys. Res., 90, 1963-1982, 1985.

Valet, J.-P., C. Laj, and C. G. Langereis, Sequential geomagnetic reversals recorded in Upper Tortonian marine clays in western Crete (Greece), J. Geophys. Res., 93, 1131-1151, 1988.

Valet, J.-P., L. Tauxe, and B. M. Clement, Equatorial and midlatitude records of the last geomagnetic reversal from the Atlantic Ocean, Earth Planet. Sci. Lett., 94, 371-384, 1989.

Valet, J.-P., P. Tucholka, V. Courtillot, and L. Meynadier, Palaeomagnetic contraints on the geometry of the geomagnetic field during reversals, Nature, 356, 400-407, 1992.

Vallarta, M. S., Theory of the geomagnetic effects of cosmic radiation, in Encyclopedia of Physics, 46/1, Ed. S. Flügge, Springer-Verlag, Berlin, Heidelberg, New York, pp 88-129 1961.

Walker, R. J., and C. T. Russell, Solar-wind interactions with magnetized planets, in Introduction to Space Physics, Eds. M.G. Kivelson and C.T. Russell, Cambridge University Press, Cambridge, pp 164-182, 1995.

Walt, M., Introduction to Geomagnetically Trapped Radiation, Cambridge University Press, Cambridge, 1994.

Weeks, R. J., M. Fuller, and I. Williams, A model for transitional field geometries involving low-order zonals and drifting nondipole harmonics, J. Geophys Res., 93, 11613-11620, 1988.

Williams, I., and M. Fuller, Zonal harmonic models of reversal transition fields, J. Geophys. Res., 86, 11657- 11665, 1981.

Willis, D. M., and A. R. Gardiner, Equations for the field lines of a sectorial magnetic multipole, Geophys. J., 95, 625-632, 1988.

Willis, D. M., and L. R. Young, Equation for the field lines of an axisymmetric magnetic multipole, Geophys. J.R. Astron. Soc., 89, 1011-1022, 1987. 